\title{
TIC e inteligencia artificial en la revisión del proceso de escritura: su uso en las universidades públicas valencianas
}

The use of ICTs and artificial intelligence in the revision of the writing process in Valencian public universities

TIC i intel.ligència artificial en la revisió del procés d'escriptura: el seu ús en les universitats públiques valencianes

\author{
Beatriz Martín-Marchante*®i
}

Departamento de lingüística Aplicada, Universitat Politècnica de València, Valencia, España

*Autor para correspondencia: beamarm2@upvnet.upv.es (Beatriz Martín-Marchante)

Recibido: 09/03/2021 | Aceptado: 08/09/2021 | Publicado: 15/01/2022

Cómo citar:

Martín-Marchante, B.

(2022). TIC e inteligencia artificial en la revisión del proceso de escritura: su uso en las universidades públicas valencianas. Research in Education and Learning Innovation Archives, 28,16-31. 10.7203/realia.28.20622

Copyright: El/La Autor/a. Open Access: Este es un artículo de acceso abierto distribuido bajo los términos de la licencia Creative Commons

Attribution-NoDerivatives 4.0 International licence (CC BY-ND 4.0)

Financiación: None informed
RESUMEN: La incorporación de las TIC y la inteligencia artificial (IA) en el ámbito educativo generó, desde sus inicios, un debate social todavía vigente. Uno de los aspectos más controvertidos es el de la introducción de los sistemas de tutoría inteligente y aprendizaje en línea. En el año 2016 el informe Artificial Intelligence and Life in 2030 pronosticaba que estos serían habituales en las aulas en tan solo quince años. Han pasado 5 años desde entonces y es preciso conocer cuál es el estado de la cuestión en estos momentos. El propósito de este estudio es, por una parte, investigar la relación entre la recomendación de uso de las TIC e inteligencia artificial en el proceso de escritura por parte del profesorado, y el uso real que de estas hacen los estudiantes. Por otra parte, se pretende averiguar qué tipo de evaluación automatizada e información de retorno correctiva aplican los docentes, así como también, qué percepción tienen ambos colectivos sobre el aprovechamiento de las TIC y la IA. Para llevar a cabo la investigación se diseñaron dos cuestionarios ad hoc al que respondieron 53 profesores y 101 estudiantes de la Universitat Politècnica de València (UPV) y de la Universitat de València (UV). Entre los resultados obtenidos cabe destacar que el 79\% de los profesores recomienda el uso de TIC, pero solo el 61,4\% del alumnado sigue dicha recomendación. Una de las conclusiones a las que se llega es que la integración completa de las TIC y la IA en la enseñanza y aprendizaje de lenguas está todavía por llegar, y que la mayoría de los participantes en este estudio admiten no aprovechar óptimamente el potencial educativo que estas ofrecen.

PALABRAS CLAVE: EAILAO; educación superior; escritura académica; inteligencia artificial; TIC

ABSTRACT: From the outset, the incorporation of ICT and artificial intelligence (AI) in the educational field generated a social debate that is still ongoing. One of the most controversial aspects was the introduction of intelligent tutoring and online learning systems. In 2016, the Artificial Intelligence and Life in 2030 report predicted that these systems would become the norm in classrooms in just fifteen years. Five years since the publication of that report, we need to know the current state of the art. One aim of this study is to investigate the relationship between the teacher-recommended use of ICT and artificial intelligence in the writing process and how this technology is actually used by students. Another aim is to determine what type of automated assessment and corrective feedback is applied by teachers and what perception both groups have about the use of ICT and AI. To conduct this study, we designed two ad hoc questionnaires, which were completed by 53 professors and 101 students from the Polytechnic University of Valencia (UPV) and the University of Valencia 
(UV). One result from this research is that while $79 \%$ of teachers recommend using ICT, only $61.4 \%$ of students follow this recommendation. One conclusion we can draw is that ICT and AI have not yet been fully integrated into language teaching and learning. At the same time, most participants in this study admit that they do not take full advantage of this technology.

KEYWORDS: ICALL; ICT; academic writing; higher education; artificial intelligence

RESUM: La incorporació de les TIC i la intel-ligència artificial (IA) a l'educació va plantejar des del principi un debat social encara vigent, un dels aspectes més controvertits del qual és el dels sistemes de tutoria intel-ligent i aprenentatge en línia. En l'any 2016, l'informe Intel-ligència artificial $i$ vida en el 2030 pronosticava que aquestes eines serien habituals en les aules en tan sols quinze anys. N'han passat cinc des de llavors i cal saber quin és l'estat de la qüestió en aquests moments. El propòsit d'aquest estudi és, d'una banda, investigar la relació entre la recomanació de l'ús de les TIC i de la IA en el procés d'escriptura del professorat i l'ús real que en fan els estudiants; i, d'una altra, esbrinar quin tipus d'avaluació automatitzada i informació de retorn correctiva utilitzen els docents, així com també, què pensen tots dos col-lectius sobre l'aprofitament de les TIC i la IA. Per fer la investigació es van dissenyar dos qüestionaris ad hoc que van respondre 53 professors i 101 estudiants de la Universitat Politècnica de València (UPV) i de la Universitat de València (UV). Entre els resultats obtinguts cal destacar que el $79 \%$ dels professors recomana l'ús de TIC, però només el $61,4 \%$ dels alumnes segueix aquesta recomanació. Una de les conclusions a les quals s'arriba és que la integració completa de les TIC i la IA en l'ensenyament i l'aprenentatge de llengües està encara per arribar i que la majoria dels participants en aquest estudi admeten que no aprofiten òptimament el potencial educatiu que aquestes ofereixen.

PARAULES CLAU: EAILAO; educació superior; escriptura acadèmica; ntel-ligència artificial; TIC

\section{Notas de aplicación práctica}

\section{Qué se sabe sobre este tema}

- La investigación acerca del uso de las TIC y la IA en el ámbito educativo se ha centrado, en los últimos años, en discernir la efectividad de estas, sus ventajas y el impacto que su uso produce en la enseñanza y aprendizaje.

- Existe también un gran volumen de estudios publicados que describen las actitudes de profesores y estudiantes hacia el uso de las TIC para el aprendizaje de lenguas. Sin embargo, encontramos un número escaso de estudios que analicen la relación existente entre el uso que el profesorado hace de las TIC y la IA y el uso que hace de estas el estudiantado.

- Tampoco hemos encontrado suficientes trabajos que investiguen sobre los propósitos, expectativas y percepciones acerca del aprovechamiento de las TIC e IA. Más concretamente, carecemos de datos actualizados respecto a la situación en las universidades públicas valencianas.

\section{Qué añade este artículo}

- El presente estudio contribuye a dilucidar las cuestiones arriba mencionadas centrándose, específicamente, en la utilización de la IA y las TIC para la redacción y revisión de trabajos académicos. Los resultados obtenidos descubren una carencia respecto al uso de sistemas de tutorización inteligentes, entre otras.

- Este trabajo ofrece, además, evidencias acerca de la percepción de aprovechamiento de las TIC y de la IA y el tipo de evaluación e información de retorno correctiva que el profesorado suele utilizar.

- Los datos que se aportan son de interés porque ofrecen una visión, cercana y actualizada, del profesorado y del estudiantado pertenecientes a distintos departamentos de la Universitat Politècnica de València (UPV) y de la Universitat de València (UV).

\section{Implicaciones para la práctica y/o política}

- Investigar y facilitar datos sobre el uso de las TIC e IA por parte de estudiantes y profesorado de educación superior, es imprescindible para mejorar la docencia y su aprovechamiento. Los resultados derivados ofrecen una visión realista que se presenta alejada de los presupuestos teóricos. 
- La interpretación de estos resultados puede contribuir a perfeccionar los criterios y parámetros establecidos por las autoridades competentes en materia de desarrollo tecnológico y digital de la UPV y de la UV.

- Esta investigación demuestra la necesidad de seguir trabajando en la elaboración de propuestas pedagógicas innovadoras tales como talleres para el desarrollo del aprendizaje autónomo dirigidos al estudiantado o la inversión en sistemas de tutoría inteligente que sirvan de apoyo al profesorado, sobre todo, en la situación actual de pandemia que ha precipitado la implementación de la docencia virtual.

\section{INTRODUCCIÓN}

El uso de la tecnología como herramienta para desarrollar las diferentes destrezas lingüísticas ha recibido una gran atención durante décadas (Chapelle, 2007; Young, 2003; Yunus, 2007) y sin lugar a duda, como afirman Chapelle y Sauro (2017, p. 1), "la tecnología se ha convertido en parte integral de la forma en que la mayoría de los estudiantes de idiomas en el mundo acceden a materiales en su segundo idioma y en lengua extranjera, interactúan con otros, aprenden dentro y fuera del aula y realizan pruebas de lengua". Sin embargo, el uso de tecnología en la enseñanza y aprendizaje de lenguas no es nada nuevo, ya que los ordenadores se han venido utilizado para la enseñanza de idiomas desde la década de los 60. En aquellos años las preocupaciones entre los investigadores estaban relacionadas, principalmente, con las actitudes de los docentes ante las nuevas tecnologías en el aula (Lee, 1970). La aparición en aquel entonces de la palabra tecnofobia reflejaba el rechazo de muchos docentes ante los nuevos avances tecnológicos y la preocupación por la falta de seguridad, de facilidades, o de formación del profesorado (Weil y Rosen, 1995). Muchos docentes argumentaban que no sabían nada de tecnología, aunque en realidad algo sí sabían, puesto que utilizaban correo electrónico, procesador de textos e Internet, como explican Dudeney y Hockly (2007). Ciertamente, hasta no hace mucho tiempo, ha existido preocupación por el grado de dominio del procesador de textos. Prueba de ello es el cuestionario diseñado por Dudeney y Hockly (2007) en el que se indaga sobre los conocimientos informáticos y de programas de tratamiento de textos de los docentes. Los ítems interrogaban acerca de la capacidad de los docentes para:

- Abrir, guardar, recuperar e imprimir documentos.

- Seleccionar texto con el teclado.

- Seleccionar texto con el ratón.

- Cortar, copiar y pegar.

- Arrastrar y soltar.

- Buscar y reemplazar.

- Usar control de cambios.

- Usar formularios.

En cuanto a la integración de las TIC, Bax (2003) se planteaba cuál era el pasado, el presente, y el futuro de tal integración y sugería que la tecnología debía volverse invisible tanto para el estudiante como para el/la docente. Argumentaba que para que pudiera producirse una integración completa de la tecnología en el proceso de aprendizaje era necesario que se convirtiera en algo natural dentro del aula, del mismo modo que los instrumentos más tradicionales, tales como el libro de texto o el diccionario. Dudeney y Hockly (2007) añadían que esta integración de las TIC no sería posible sin la formación del profesorado. Pronosticaban que el cambio se produciría 
de manera variable, y que no habría una gran permutación o tendencia, sino más bien una variedad de tendencias, algunas más rápidas y otras más lentas. También adelantaban que habría grupos de profesores que abrazarían las nuevas tecnologías mientras que otros se mantendrían impasibles ante los cambios tecnológicos. Sus predicciones parecen haberse cumplido en gran medida, y el futuro del que hablaban entonces es ya nuestro presente. El cuestionario de Dudeney y Hockly (2007) puede carecer de sentido en el momento actual, ya que pertenece a una fase del pasado ya superada; de hecho, la investigación acerca de las TIC en las aulas se ha centrado, en las últimas décadas, en discernir su grado de efectividad, sus ventajas (Muehleisen, 1997; Torres, 2006; Warschauer y Kern, 2000a), su uso pedagógico o el impacto que pueda tener en la enseñanza y aprendizaje (Zawacki-Richter, Marín, Bond, y Gouverneur, 2019). En estos momentos, sin embargo, necesitamos seguir investigando qué ha sucedido en los últimos años. Este trabajo indaga sobre el estado de la cuestión y el grado de implicación tanto de profesores como de estudiantes en la integración de las TIC y la IA en el ámbito educativo, en concreto, en la enseñanza y aprendizaje de lenguas. Las preguntas de investigación que impulsaron este estudio se plantearon tras observar que muchos de nuestros estudiantes hacían poco o nulo uso de determinadas herramientas TIC para la realización de sus trabajos escritos. Surgió, así, la pregunta de si este hecho podía deberse a la falta de recomendación de su uso por parte del profesorado. Al mismo tiempo, emergió el interés por averiguar qué tipo de evaluación e información de retorno aplica normalmente el profesorado para la corrección de errores. Por otra parte, se quiso averiguar qué percepción tenían ambos colectivos sobre el grado de aprovechamiento de las TIC y la IA durante el proceso de producción escrita.

\section{MARCO TEÓRICO}

En las últimas décadas, la integración y las aplicaciones de las TIC han influido en la manera de enseñar y de aprender cualquier idioma. Las TIC ha incorporado una dimensión social que permite lo que se denomina "aprendizaje en red" como señalan Kannan y Munday (2018). Este hecho ha revitalizado los marcos teóricos anteriores del aprendizaje de lenguas asistido por ordenador (ALAO), más conocido por sus siglas en inglés CALL ${ }^{1}$, y del aprendizaje de lenguas asistido por dispositivos móviles o MALL. Como es lógico, también la forma en que se han utilizado las TIC ha ido cambiando a lo largo de los años. Thu-Dang (2013) distingue varias formas de utilizar las TIC: como herramienta de localización y recuperación, como herramienta de interacción, y como instrumento para la creación de materiales. Gracias a su uso como herramienta de localización y recuperación, los profesores pueden buscar en Internet los materiales de aprendizaje disponibles para sus clases, y acceder a diferentes tipos de recursos digitales como libros electrónicos, imágenes, audios y videos, etc. El modelo SAMR de Puentedura (2013) también se puede utilizar para clasificar y evaluar la integración de las TIC, ya que como indican Nhu, Keong, y Wah (2018), proporciona un marco apropiado que consta de cuatro clasificaciones del uso de la tecnología para las actividades de aprendizaje: "sustitución, aumento, modificación, y redefinición". En la primera, la tecnología sustituye a otras actividades de aprendizaje sin cambios funcionales. En la segunda, la tecnología proporciona un sustituto de otras actividades de aprendizaje, pero con mejoras funcionales. En las actividades de "modificación", la tecnología permite rediseñar la actividad de aprendizaje, mientras que en las de "redefinición", la tecnología permite la creación de tareas que no se podrían haber hecho sin el uso de la tecnología.

\footnotetext{
${ }^{1} 1$ En este artículo utilizaremos las siglas en inglés: CALL e ICALL, en lugar de ALAO e EAILAO respectivamente, por ser más conocidas y utilizadas en la literatura de este campo de estudio.
} 
En cuanto a la evolución del aprendizaje basado en tecnología a lo largo del tiempo, se distinguen cuatro pedagogías o entornos de aprendizaje para la enseñanza de la gramática de la L2: CALL tutorial, CALL inteligente o ICALL, aprendizaje basado en datos (DDL) y comunicación mediada por computadora (CMC), como indican Heift y Vyatkina (2017). En estos entornos, el ordenador asume el papel de una herramienta, según Levy (1997). Además de las herramientas más completas del tipo ICALL, los estudiantes también pueden tener acceso a diccionarios en línea (Hamel, 2010), así como también a correctores gramaticales y ortográficos (Burston, 2001; Lee, 2014). En cuanto a los avances que se están produciendo en el campo de CALL, actualmente, el más importante es la inclusión de un número cada vez más significativo de sistemas que integran y utilizan las técnicas de la inteligencia artificial (AI) dando lugar a la ICALL. Lavilla (2016) explica cómo los sistemas de tutoría inteligente, o el de reconocimiento automático de habla (RAH) y el procesamiento de lenguajes naturales (PLN), ha facilitado el desarrollo de los sistemas de tutoría inteligente, que han pasado rápidamente del laboratorio al uso real. Estos tutores cognitivos imitan el rol del profesor y guían el aprendizaje y la práctica en distintas disciplinas. Además, dan pistas a los estudiantes cuando se bloquean, les proporcionan información de retorno inmediata, e incluso diseñan secuencias de aprendizaje personalizadas para cada alumno. La autora (ibid.) pone como ejemplo Duolingo, pero existen ya numerosas aplicaciones enfocadas al aprendizaje de lenguas extranjeras, que detectan los errores del estudiante, los corrigen y le ayudan a progresar a su ritmo. Es necesario puntualizar, llegados a este punto, que, aunque la ICALL y la CALL tradicional pueden parecer similares, desde el punto de vista del usuario no lo son, ya que como señala Araujo (2013), los programas tradicionales de CALL piden al alumno que dé una respuesta de entre un número limitado, mientras que los programas ICALL admiten un conjunto de enunciados nuevos e intentan descifrar lo que quiere decir el alumno o las intenciones de este. Con ello se puede facilitar la información de retorno o bien organizar actividades complementarias o adicionales. El tipo de información de retorno que ofrecen la ICALL y la CALL también es diferente. Los programas CALL ofrecen respuestas del tipo correcto/incorrecto, mientras que las de ICALL tiene un carácter más cualitativo, llamando la atención sobre aspectos que resultan problemáticos y proponiendo soluciones o medidas a tomar para solucionar los problemas que se detectan.

\section{1. ¿Qué es la inteligencia artificial?}

La IA ha estado entre nosotros desde hace más de 50 años, pero fue en la década de los 80 cuando una serie de investigadores llevaron a cabo estudios sobre la eficacia del procesador de textos. En aquel entonces, como explica MacArthur (2009), los resultados obtenidos apoyaron un principio general, el de que la tecnología y las herramientas por sí mismas tienen poco impacto en el aprendizaje, especialmente en el de los estudiantes con dificultades. Más bien, subraya el autor (ibid.), el aprendizaje depende de una combinación de tecnología e instrucción diseñada para ayudar a los estudiantes a aprovechar las capacidades de la tecnología. Sin embargo, otros estudios sí demostraron la efectividad de un modelo de instrucción de escritura que incluía procesamiento de textos y estrategias diseñadas para aprovechar las capacidades de este. El procesamiento de textos es un aspecto de la tecnología de escritura que cuenta con el respaldo de una base de investigación sustancial (Dalton y Hannafin, 1987; Frase, Kiefer, Smith, y Fox, 1985; Wolfe, Bolton, Feltovich, y Bangert, 1996). Los metaanálisis de estudios que compararon la enseñanza de la escritura con y sin procesamiento de texto han encontrado efectos positivos moderados en la calidad de los trabajos escritos con efectos más importantes para los estudiantes de bajo rendimiento. MacArthur (2009) explica que el procesador de textos era una aplicación prometedora porque los profesores podían integrarlo con relativa facilidad para mejorar la revisión de los textos de los discentes. Por tanto, se puede afirmar que el uso del procesador de textos en 
la enseñanza de la escritura tiene un efecto positivo moderado en producción escrita de los estudiantes (Dudeney y Hockly, 2007). Otros investigadores (Ferris y Roberts, 2001; Sheppard, 1992) han informado, además, sobre las ventajas de alentarles a revisar sus textos escritos inmediatamente después de recibir información de retorno sobre su escritura, y sostienen que esta estrategia les ayuda a mejorar la calidad de sus redacciones.

González-Rodríguez y Álvarez Mosquera (2020), por su parte, concluyen que, aunque tanto la información de retorno, por un lado, como la instrucción explícita, por otro, ayudan a mejorar la competencia retórica de los estudiantes en sus escritos académicos, solo el tratamiento combinado de ambas variables da como resultado una mejora estadísticamente significativa. A todo esto, habría que añadir que, como sugiere Lee (2014), la información de retorno también debe ser percibida por los estudiantes como una experiencia de aprendizaje significativa y relevante que les haga conscientes de sus fortalezas y debilidades y les ayude a remediar sus dificultades de escritura.

También Araujo (2013) defiende la utilidad de los ordenadores a la hora de redactar, puesto que hay programas que incluso ayudan a los alumnos en la fase de preparación de un ejercicio de expresión escrita, es decir, a la hora de generar y presentar ideas. No hay que olvidar que la mayoría de los procesadores de texto incluye correctores ortográficos y diccionarios y que muchos diccionarios incluyen una sección de referencia que ofrece aclaraciones gramaticales, audio o vídeos para el reconocimiento de palabras, y para mostrar su pronunciación y su uso en el contexto adecuado. En definitiva, se les facilita muchísima información lingüística, así como las herramientas para estudiar la lengua.

En definitiva, los asistentes de escritura basados en el PNL y en el aprendizaje automático ayudan a los usuarios en el proceso de producción escrita, ya que corrigen los errores gramaticales dentro de un texto escrito, brindan recomendaciones para mejoras posteriores y ofrecen recursos adicionales para completar el conocimiento y seguir estudiando. Como explica Pokrivcakova (2019), en el aula de lengua extranjera, estos sistemas ayudan a los alumnos a pasar por el proceso de escritura de forma individual, a corregirse, y a pensar en el proceso en sí. El uso de la IA facilita, de esta manera, la autorregulación y la autonomía del alumno. Algunos ejemplos de asistentes de escritura de IA son: Grammarly, ProWriting Aid, Textio, AI Writer, Textly AI y Essaybot.

Como se ha visto, los sistemas de tutoría inteligente y de evaluación automatizada ofrecen numerosas ventajas y a muchos de ellos se accede con tan solo un clic. Esto nos podría llevar a pensar que su uso esté siendo generalizado, sin embargo, no tenemos datos al respecto. Ha habido un gran volumen de estudios publicados que describen las actitudes de profesores y estudiantes hacia el uso de las TIC para el aprendizaje de lenguas. No obstante, se precisa más investigación que indague sobre qué tipo de herramientas se recomiendan y se utilizan para la realización de trabajos académicos y sobre la percepción de aprovechamiento que se tiene de estas. Así pues, el propósito del presente estudio es abordar estas cuestiones, ya que hemos encontrado pocos estudios actualizados que revelen el uso real que se hace de ellas en la enseñanza y aprendizaje de lenguas en el contexto de la educación superior y dentro de nuestro ámbito territorial.

\section{MÉTODO}

Los objetivos fundamentales de este trabajo son, por una parte, describir y comparar el tipo de herramientas TIC e IA más recomendadas por el profesorado de lenguas de la UPV y de la UV y ver si el alumnado sigue sus recomendaciones. Se quiere, también, averiguar qué tipo de evaluación e información de retorno aplica normalmente el profesorado. Por otra parte, se quiere investigar la percepción de ambos grupos 
sobre el grado de aprovechamiento de las TIC y la IA. Por lo tanto, las preguntas de investigación que impulsaron el presente estudio son las siguientes:

- ¿Existe relación entre la recomendación de uso de TIC e IA por parte del profesorado y el uso real que hacen los estudiantes de estas?

- ¿Qué tipo de evaluación automatizada e información de retorno correctiva aplica el profesorado?

- ¿Qué percepción tiene el profesorado y el alumnado sobre el aprovechamiento de las TIC y la IA?

\subsection{Sujetos y materiales}

La población objeto del estudio está compuesta por docentes y estudiantes. Para dar respuesta a las preguntas de investigación se obtuvo información a través de dos cuestionarios ad hoc utilizando la herramienta Google Forms. El estudio se realizó durante el curso académico 2019- 2020. Los cuestionarios, uno para cada colectivo, estuvieron abiertos y disponibles desde septiembre de 2019 hasta noviembre de $2020 \mathrm{y}$ fueron cumplimentados por un grupo de 101 estudiantes y un grupo de 53 profesores de lenguas de la Universitat Politècnica de València (UPV), y de la Universitat de València (UV). El criterio empleado para la selección de los participantes fue que en todos los casos ambos grupos contasen con apoyo técnico suficiente y acceso completo a Internet y las TIC, independientemente del curso en el que estuviesen. El $40 \%$ de los participantes estudian exclusivamente inglés, un $27 \%$ sólo inglés técnico y un $17 \%$ alemán. Un $14 \%$ está aprendiendo más de una lengua simultáneamente. La edad media de la muestra de estudiantes es de 19,4 y la de docentes es de 61 años. El 57,4\% de los respondientes del grupo de estudiantes son de género masculino mientras que el $72 \%$ del profesorado es de género femenino.

En los cuestionarios, de carácter mixto, se distinguieron dos partes: una en la que se incluyeron cuatro preguntas sobre datos demográficos tales como edad, sexo, asignatura (francés, alemán, catalán e inglés) y curso, y otra en la que se incluyeron 12 ítems de respuesta corta, de respuesta cerrada politómica, ya que permiten un análisis estadístico descriptivo más rápido, y otros ítems de escala Likert para medir las actitudes. Además, se incluyó una pregunta de respuesta abierta para ofrecer a los participantes la posibilidad de añadir comentarios relacionados con el tema del cuestionario.

Los ítems del cuestionario fueron seleccionados atendiendo a los temas propuestos en la bibliografía especializada y las preguntas de investigación (ver el Apéndice A). Algunos de los ítems no se incluyen aquí por no corresponder a los objetivos específicos planteados en este estudio.

A continuación, se muestra un resumen de preguntas de los cuestionarios dirigidas tanto al profesorado como al alumnado:

- ¿Recomiendas / utilizas algún recurso o herramientas TIC para hacer tus trabajos escritos en la clase de lengua extranjera u otras lenguas?

- ¿Qué herramientas recomiendas normalmente?

- ¿Qué técnica de feedback y corrección de errores de escritura de tus alumnos utilizas habitualmente?:

- ¿Cuántos de tus alumnos/compañeros crees que saben sacar partido al uso de las TIC?

- ¿En qué porcentaje crees que saben sacar partido al uso de las TIC en el ámbito educativo? 
- ¿Deseas añadir algo más relacionado con el tema del cuestionario?

Para garantizar la confiabilidad del cuestionario, se recurrió al juicio de expertos sobre una prueba piloto llevada a cabo con 50 informantes voluntarios, ya que no se encontró un cuestionario previamente validado que abordase las preguntas de investigación aquí trazadas. Como resultado de ello, los 30 ítems iniciales quedaron reducidos a 16, y se modificó la redacción de algunos ítems para que resultasen más comprensibles. Antes de cumplimentar el cuestionario los participantes fueron informados sobre las características de este, y firmaron un consentimiento en el que se les informaba sobre su carácter voluntario, la no relación con los contenidos y la evaluación de su asignatura, así como también, la posibilidad de resolver dudas durante su realización.

\subsection{Muestra y metodología estadística}

Para dar respuesta a las preguntas de investigación se utilizaron dos muestras independientes obtenidas a partir de sendos cuestionarios auto cumplimentados sobre las poblaciones de profesores y alumnos. Para estimar una proporción poblacional a partir de la muestra de 53 profesores, se tiene un error máximo del 8,7\% para $\mathrm{p}=\mathrm{q}=50$ (peor de los casos posibles), confianza del $95 \%$ y asumida población de tamaño $\mathrm{N}=90$.

Análogamente, para estimar una proporción poblacional a partir de la muestra de 101 alumnos, se tiene un error máximo del $9,2 \%$ para $\mathrm{p}=\mathrm{q}=50$ (peor de los casos posibles), confianza del $95 \%$ y asumida población de tamaño $\mathrm{N}>1.000$.

Por una parte, se ha realizado un análisis descriptivo que proporcionó los estadísticos más relevantes para todas las variables recogidas en los dos formularios: porcentajes y frecuencias absolutas y relativas, al tratarse de valoraciones en escala categórica.

Por otra parte, se ha llevado a cabo un análisis inferencial que tiene por objeto estudiar las diferencias en las respuestas del grupo de profesores y alumnos en aquellas preguntas con los mismos contenidos. Para ello se ha empleado el test no paramétrico de Mann-Whitney para determinar si la distribución de las respuestas de preguntas comunes (o comparables) en ambos cuestionarios es o no similar. Es el caso de las herramientas TIC recomendadas por profesores vs. utilizadas por alumnos y de la percepción del nivel de aprovechamiento de las TIC. También se ha empleado el test Chi2 de Pearson para evaluar la homogeneidad de la tasa de uso de un tipo concreto de herramienta en las dos muestras. Se justifica su aplicación por el hecho de que muchas de las preguntas sobre uso de herramientas son de respuesta múltiple y es necesario dicotomizar a variables binarias (sí/no usa). En el caso de una alta proporción de frecuencias esperadas bajas, se ha utilizado el test exacto de Fisher. El nivel de significatividad empleado en los análisis ha sido el $5 \%(\alpha=0.05)$.

En cuanto al tamaño de efecto de la muestra se tiene que un test $\mathrm{Chi}^{2}$ alcanza una potencia del $86,6 \%$ para detectar como significativamente distintas proporciones $90 \% \mathrm{y}$ $70 \%$ en la recomendación/uso de una herramienta TIC por parte de los $\mathrm{n}=53$ profesores y $\mathrm{n}=101$ alumnos de la muestra actual. Calculado para un nivel de confianza del $95 \%$.

\section{ANÁLISIS Y RESULTADOS}

Los resultados de este estudio muestran que, a pesar de que más de la mitad (54,7\%) de los profesores entrevistados recomienda siempre la utilización de herramientas y recursos TIC para la realización de los trabajos escritos, sólo el 13,9\% de los alumnos declara utilizarlas siempre. El siguiente gráfico (Figura 1), proporciona una visualización conjunta de las preguntas P1 y A3 de los cuestionarios de profesores y alumnos respectivamente:

- P1 ¿Recomiendas a tus alumnos la utilización de algún recurso o herramientas TIC/IA para hacer sus trabajos escritos en la clase de lengua extranjera u otras 
lenguas?

- A3 ¿Utilizas TIC/IA para tus trabajos académicos escritos?

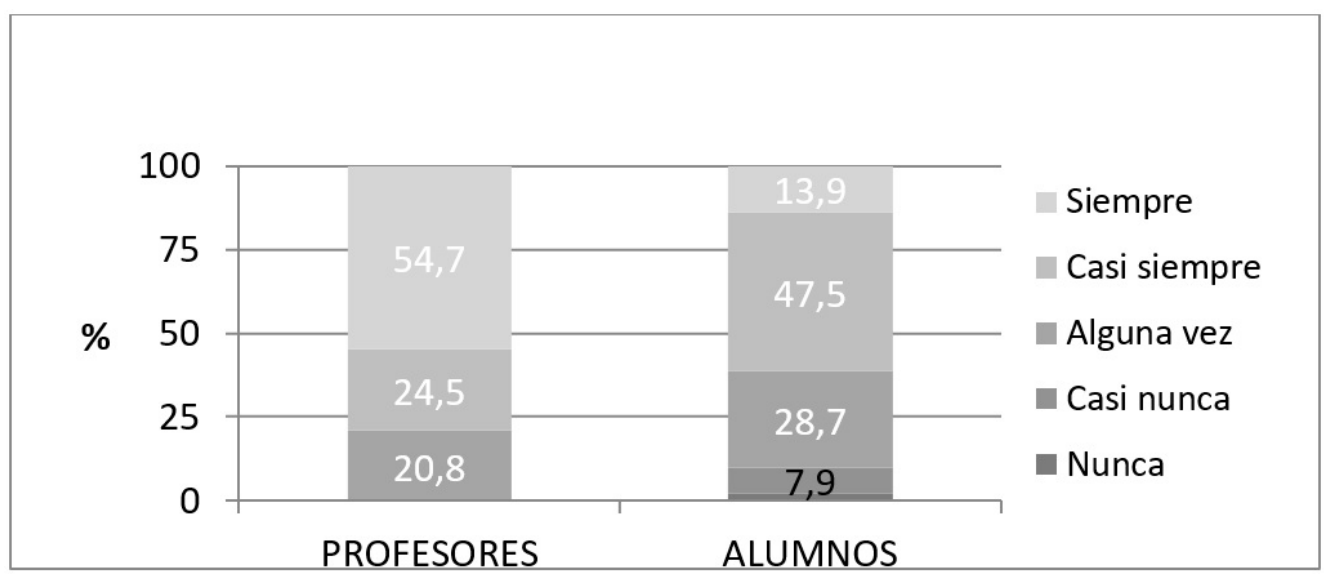

Figura 1. Recomendación por profesores y utilización por estudiantes de TIC

Si consideramos las dos categorías de máxima frecuencia (siempre y casi siempre), la heterogeneidad disminuye. El 79,2\% de los profesores las recomiendan habitualmente, frente al $61,4 \%$ de alumnos que las usan. Se ha realizado una prueba estadística para evaluar si las distribuciones de frecuencias observadas en profesorado y alumnado son o no similares. El resultado es estadísticamente significativo ( $<<0,001$, test de MannWhitney): la tasa de recomendación por parte de los profesores es significativamente más elevada que la tasa de utilización por los alumnos.

En cuanto a las herramientas específicas recomendadas y utilizadas por profesorado y alumnado respectivamente (Figura 2), son cuestiones que se plantean en las preguntas P2 ("Herramientas que recomiendas normalmente") y A4 ("herramientas que utilizas normalmente”) de profesorado y alumnado respectivamente:

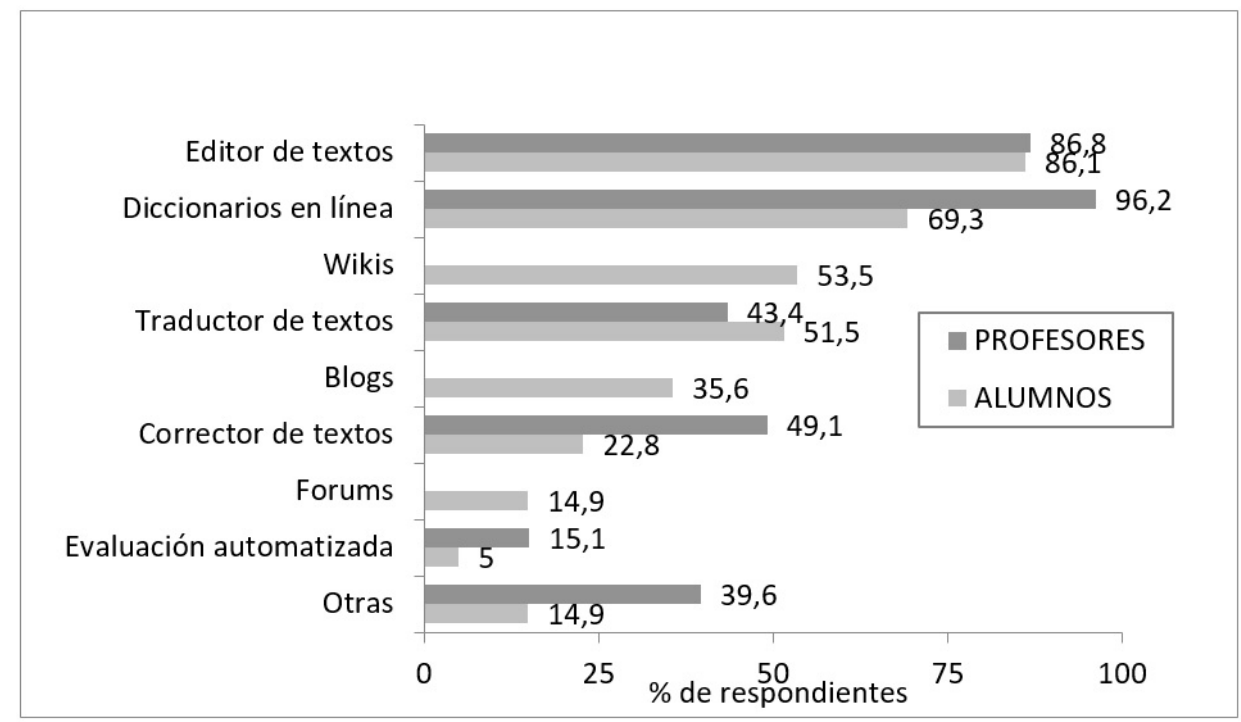

Figura 2. Herramientas más recomendadas por el profesorado y utilizadas por el alumnado.

Respecto a las respuestas que sugirieron de forma común a ambos grupos, referidas al tipo de TIC e IA utilizadas (P3 y A3), destaca el mayor grado de recomendación por 
parte de los profesores de los diccionarios, los correctores de textos y 'otras' herramientas. Wikis, blogs y fórum, fueron opciones que se plantearon exclusivamente a los alumnos de manera que, si se calcula el porcentaje de alumnos que citan alguna/s de estas opciones u 'otras' no especificadas, se obtiene un $67,3 \%$, por lo que el gráfico anterior podría replantearse así (ver Figura 3).

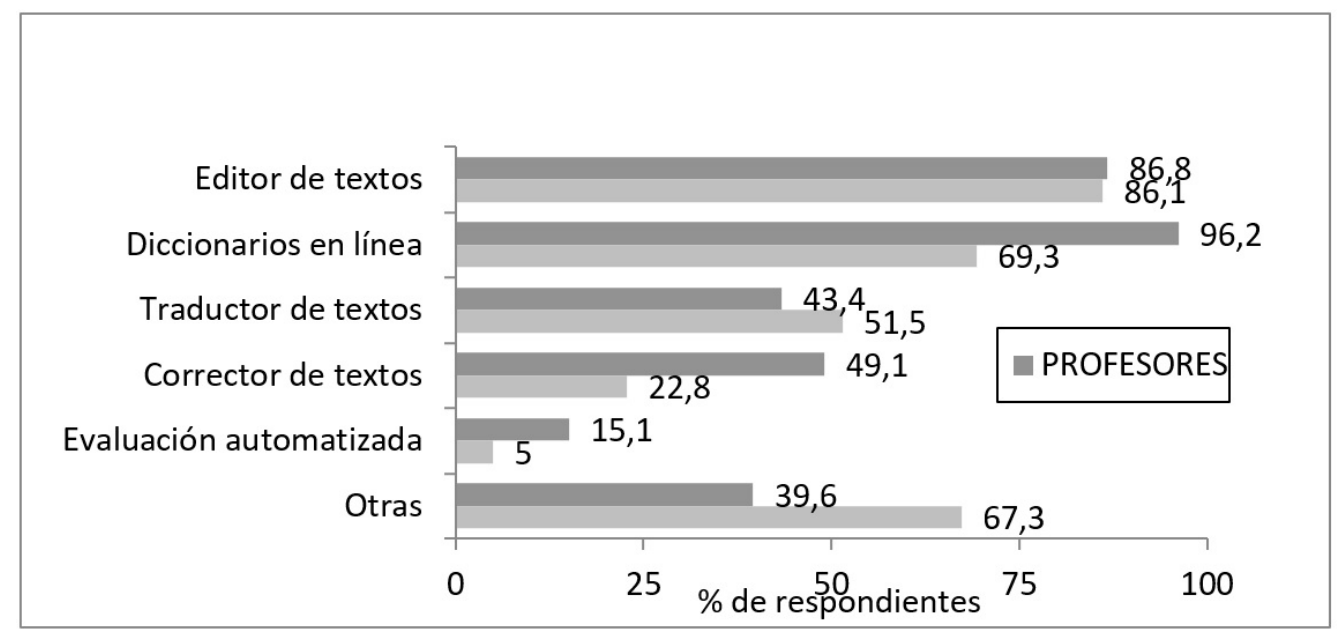

Figura 3. Herramientas más recomendadas por los profesores y utilizadas por los alumnos.

Se observa, también, que la utilización de la evaluación automatizada o de STI es muy baja en ambos grupos, ya que solo un $15 \%$ del profesorado la utiliza y en caso del alumnado el porcentaje baja hasta apenas un $5 \%$.

Las pruebas siguientes (Tabla 1) evalúan si las tasas de recomendación/uso de cada tipo de TIC son similares en profesores y alumnos:

Tabla 1. Tasa de recomendación/utilización por profesores/alumnos de diferentes

\begin{tabular}{ll} 
herramientas TIC: resultados test Chi2 & p-valor \\
\hline Editor de textos & 0,911 \\
Diccionarios en línea & $<0,001^{* * *}$ \\
Traductor de textos & 0,340 \\
Corrector de textos & $\mathbf{0 , 0 0 1 * *}$ \\
Evaluación automatizada & $\mathbf{0 , 0 3 1 *}$ \\
Otras & $\mathbf{0 , 0 0 1 * *}$ \\
\hline$*$ p $<0,05 ; * * p<0,01 ; * * * p<0,001$ &
\end{tabular}

La conclusión derivada es que los profesores recomiendan herramientas como los diccionarios, corrector de textos y evaluación automatizada, en proporción significativamente superior a la tasa de uso por los alumnos. Por otra parte, podemos observar homogeneidad en lo que respecta a editores y traductores de textos. Sin embargo, los alumnos reconocen utilizar en mayor medida una diversidad más amplia de opciones diferentes a las sugeridas (entre las que se incluyen las wikis, blogs y fórums).

En cuanto a las técnicas de información de retorno correctiva y el tipo de evaluación utilizada (P4 y A4), destaca sobre las demás, la corrección explícita y metalingüística, seguida de la implícita. La autocorrección del alumno es implementada por el 58\% del profesorado mientras que el tipo de evaluación menos utilizada es la realizada entre pares (Figura 4). 


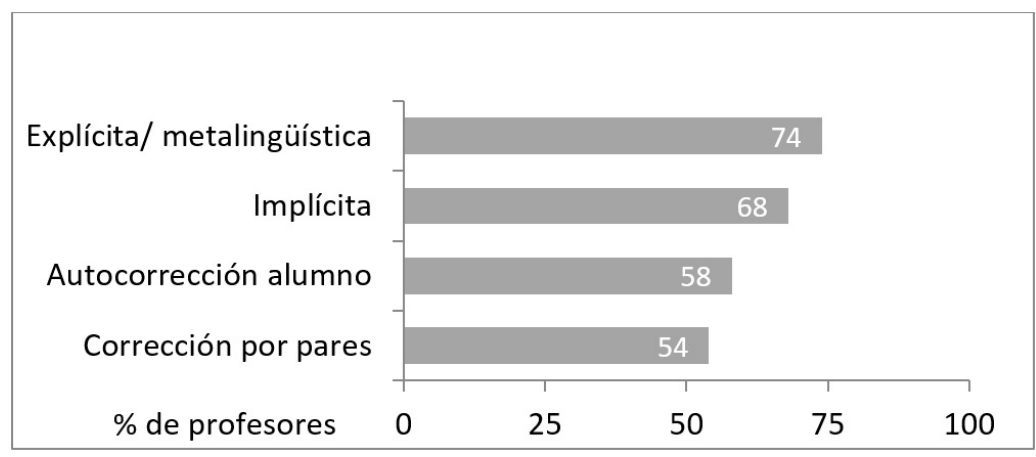

Figura 4. Técnicas de corrección e información de retorno utilizadas por los profesores.

Como se puede observar, existe cierta discrepancia con las respuestas aportadas por el alumnado, sin embargo, ambos grupos coinciden en que la técnica utilizada mayoritaria y principalmente es la de tipo explícita y metalingüística (Figura 5).

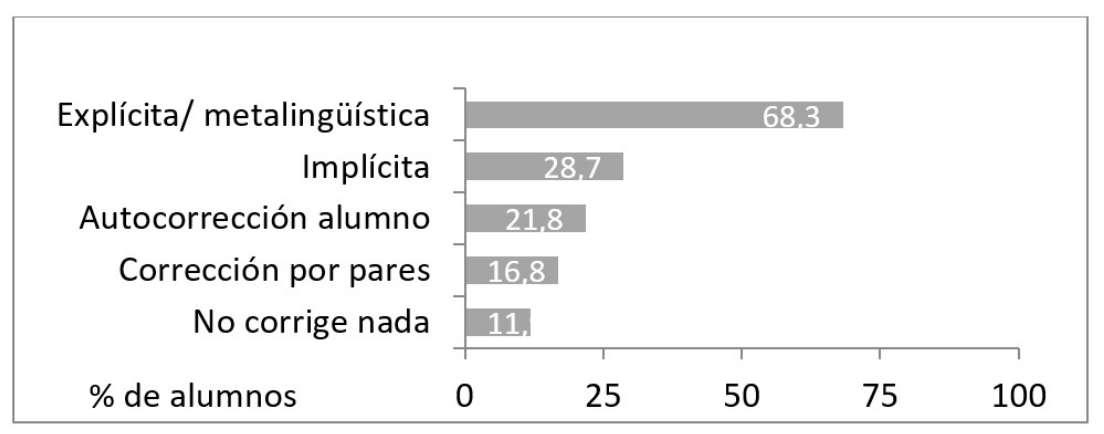

Figura 5. Técnicas de corrección e información de retorno utilizadas por los profesores, según el alumnado.

Respecto al grado de aprovechamiento de las TIC (P5, P6 y A5, A6), los profesores estimaron el porcentaje de sus alumnos que saben sacar provecho de ellas, mientras los alumnos hicieron la misma estimación respecto a sus compañeros de clase. Los resultados se resumen gráficamente en la Figura 6.

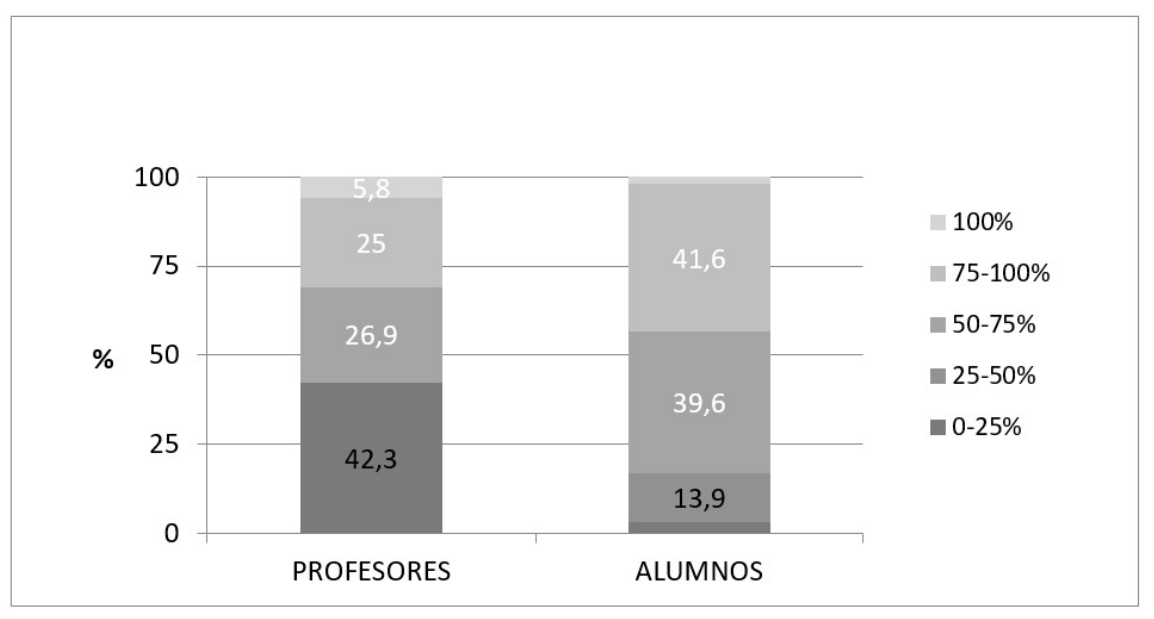

Figura 6. Porcentaje de alumnos/compañeros que saben sacar provecho de las TIC/IA

La discrepancia es manifiesta. Un $42,3 \%$ de profesores estima en menos de la cuarta parte la proporción de sus alumnos que aprovechan las TIC; pero sólo un 3\% de alum- 
nos están proporcionando ese rango para la estimación. En el extremo opuesto, un $30,8 \%$ de profesores estima en más de tres cuartas partes, los alumnos que obtienen bastante-mucho rendimiento de las TIC, frente al $43,6 \%$ de alumnos cuando opinan respecto a sus compañeros de clase. Se ha realizado una prueba estadística para evaluar si las distribuciones de rangos observadas en profesores y alumnos son o no similares y el resultado es estadísticamente significativo ( $p=0,002$, test de Mann-Whitney): la percepción de aprovechamiento de las TIC por el alumnado es significativamente más baja entre los profesores.

\section{DISCUSIÓN}

Los procesadores de texto, correctores de ortografía y gramática, programas de publicación de escritorio y concordantes permiten al alumnado utilizar o comprender el lenguaje (Warschauer y Kern, 2000b). Por este motivo es necesario, como aconseja MacArthur (2009), que los estudiantes aprendan a evaluar su escritura, a revisarla de manera efectiva y a publicarla en una variedad de formatos utilizando este tipo de herramientas que son ejemplos básicos de IA. Los beneficios de la IA parecían tan evidentes que, algunos expertos anticiparon que, en el ámbito educativo, la IA iba a crecer un $43 \%$ en el período comprendido entre 2018-2022. También el Informe Horizon 2019 Higher Education Edition (Alexander et al., 2019) predecía un crecimiento de las aplicaciones de IA relacionadas con la enseñanza y el aprendizaje incluso mayor que este porcentaje (Zawacki-Richter et al., 2019) . En el inicio de este estudio nos planteábamos cuál era el estado de la cuestión al respecto en las dos universidades públicas de Valencia. La respuesta a ello la ofrecen los datos aquí presentados, según los cuales podemos afirmar que, en cuanto a la integración de la IA en las aulas de lenguas de la UV y de la UPV, queda todavía bastante camino por recorrer, ya que los resultados obtenidos en este trabajo indican que solo un $5 \%$ utiliza la evaluación automatizada y un $22,8 \%$ los correctores de texto, por ejemplo. Este dato es, además, coherente con el resultado obtenido respecto a la preferencia de tipo de evaluación e información de retorno correctiva, el cual informa que más del $68 \%$ de los docentes utilizan la corrección explícita y metalingüística para indicar los errores cometidos por el estudiantado, siendo mucho menos utilizada (22\%) la "autocorrección" del alumno, para la cual sería necesaria la utilización de algún tipo de sistema de tutorización inteligente (STI). La relevancia y necesidad de los STI se justifica aún más en la modalidad de evaluación formativa no presencial ya que la información de retorno al estudiante, feedback, de calidad, es como explica Aretio (2021), "un respaldo permanente a la reconducción hacia la mejora".

Por tanto, podría decirse que los dos colectivos participantes en este estudio continúan en la que se ha venido en llamar "fase CALL", ya que la "fase ICALL", según las evidencias, no está todavía asentada en la práctica. Esta situación ha quedado demostrada, también, en otros estudios similares como el llevado a cabo por Romero, Carrió, y Yang (2016). En su trabajo queda de manifiesto que, aunque la enseñanza universitaria basada en las TIC se corresponde con las preferencias y el perfil del alumnado, todavía queda por conseguir que el uso que el alumnado hace de la TIC se vincule más estrechamente a la actividad de aprendizaje, ya que se ha comprobado que la mayoría de los estudiantes utilizan las TIC, única y/o principalmente para navegar por Internet. También un estudio realizado por Mangisch y Mangisch-Spinelli (2020) en la Universidad Católica de Cuyo (Argentina), concluye que el $50 \%$ de los docentes y el $49 \%$ de los alumnos dicen utilizar los teléfonos inteligentes para hablar y mandar mensajes por WhatsApp y solo el $16 \%$ del profesorado dice usarlo para fines pedagógicos. En el presente estudio ha quedado evidenciado que, aunque más de la mitad (54,7\%) de los profesores entrevistados recomienda "siempre" la utilización de herramientas y recursos TIC para la realización de los trabajos escritos, solo el 13,9\% 
del alumnado declara utilizarlas "siempre". También es motivo de reflexión el hecho de que la percepción de aprovechamiento de las TIC por parte de los dos colectivos participantes no sea tan alta como sería deseable.

Coincidiendo con Salaberry (2001), pensamos que una de las principales barreras para la implementación de tecnología percibida por los docentes es el desajuste entre las TIC, los planes de estudio existentes y el marco de tiempo de clase, como se confirma también en el estudio llevado a cabo por Albirini (2006).

\section{CONCLUSIONES}

Una de las conclusiones derivadas de los datos obtenidos en este estudio es que los profesores recomiendan el uso de herramientas TIC e IA para la redacción de trabajos escritos en proporción significativamente superior a la tasa de uso de los alumnos.

Se ha visto, también, que entre las herramientas más utilizadas por el profesorado para la redacción de trabajos escritos destacan, en primer lugar, el uso de editores de textos, seguido de diccionarios en línea y, en tercer lugar, los correctores de textos. El alumnado, por su parte, coincide con el profesorado en que las herramientas más utilizadas son los editores de texto y en segundo lugar los diccionarios en línea. Sin embargo, la tercera herramienta más utilizada por ambos grupos difiere, ya que en el caso del profesorado se trata del corrector de textos mientras que en el caso del alumnado es la de traductor de textos. Por otra parte, ambos grupos coinciden al declarar su escasa utilización de sistemas de tutorización inteligente o evaluación automatizada. Otro aspecto importante que cabe destacar es que un alto porcentaje de los participantes en esta investigación admiten no estar aprovechando al máximo las posibilidades que las TIC y la IA pueden brindar.

Para llevar a cabo este estudio no se pudo encuestar a la totalidad del alumnado y del profesorado de las asignaturas de lenguas de las universidades públicas valencianas y tampoco se emplearon datos de observación en el aula para la triangulación, pero este trabajo ofrece una visión panorámica del tema que puede establecer un punto de partida para trabajos posteriores de carácter mixto que completen los datos que se han presentado aquí.

A pesar de las limitaciones de esta investigación, el análisis e interpretación de los resultados obtenidos permite sugerir que todavía quedan algunos problemas por superar y cuestiones por resolver que pueden ser planteadas en futuras líneas de investigación. Una de estas líneas consistiría en estudiar las posibles causas que subyacen la baja frecuencia de uso de STI o evaluación automatizada para el aprendizaje de lenguas. Sería interesante averiguar hasta qué punto el enfoque comunicativo, que desestima de la corrección lingüística en favor de la fluidez, puede influir en la baja frecuencia de uso de este tipo de herramientas TIC ya que actualmente están disponibles y ofrecen múltiples posibilidades para la corrección de errores léxicos, gramaticales y /o de estilo.

Aunque las implicaciones de los resultados que se han obtenido se circunscriben a un ámbito territorial específico, el de la Comunidad Valenciana, probablemente podrían ser extrapolables a otras comunidades autónomas. Para corroborar esta conjetura se requiere, no obstante, de más estudios locales y /o nacionales que nos permitan comparar y establecer coincidencias y divergencias si las hubiere.

Para finalizar, en vista de la ubicuidad de la tecnología en la vida del alumnado y del profesorado, sería beneficioso que quienes tienen la potestad de tomar decisiones promuevan la formación digital específica, tanto para el estudiantado como para el profesorado. Igualmente, sería conveniente la inversión en sistemas de tutoría inteligente, y la involucración efectiva de ambos colectivos en el proceso de integración de las TIC y la IA para hallar, así, formas de superar las barreras percibidas. 


\section{A. APÉNDICE}

\section{ÍTEMS DE LOS CUESTIONARIOS}

Ítems relacionados con las preguntas de investigación correspondientes al cuestionario dirigido al profesorado:

- P1- ¿Recomiendas a tus alumnos la utilización de algún recurso o herramientas TIC para hacer sus trabajos escritos en la clase de lengua extranjera u otras lenguas?: Nunca- Casi nunca- Alguna vez- Casi siempre- Siempre

- P2- ¿Qué herramientas recomiendas normalmente? Puedes clicar más de una opción: Herramienta de evaluación automatizada (Write and Improve...)- Editor de textos (Word etc.)- Correctores (Grammarly etc.)- Traductor de textos- Diccionarios en red- Otras-

- P3-Indica cuáles de las anteriores en concreto (Google translator, Wordreference...)

- P4-Qué técnica de feedback y corrección de errores de escritura de tus alumnos utilizas habitualmente?: -No corrijo porque creo que no es eficaz y no encaja en mi método de enseñanza- Corrección explícita y / o metalingüística - Corrección implícita (pistas, código de corrección...)- Autocorrección del estudiante- Corrección entre pares

- P5- ¿Cuántos de tus alumnos crees que saben sacar partido al uso de las TIC?: $0-25 \%, 25-50 \%, 50-75 \%, 75-100 \%, 100 \%$

- P6- ¿En qué porcentaje crees que saben sacar partido al uso de las TIC en el ámbito educativo?: 0-25\%- 25-50\%- 50-75\%- 75-100\%- $100 \%$

- P7- ¿Deseas añadir algo más relacionado con el tema del cuestionario?

Los siguientes son algunos de los ítems que conformaron el cuestionario dirigido al alumnado:

- A1- ¿Utilizas algún recurso o herramienta TIC para la realización de tus trabajos académicos escritos?: - nunca- casi nunca - alguna vez -casi siempre - siempre

- A2- ¿Cuáles de las siguientes herramientas utilizas para realizar tus trabajos académicos escritos? Puedes clicar más de una opción: -Herramienta de evaluación automatizada Editor de textos (Word etc.) - Correctores (Grammarly etc.) - Traductor de textos- Diccionario en línea- Fórums- Wikis- Blogs- Otros

- A3-Indica cuáles de las anteriores en concreto (Google translator, Wordreference...)

- A4- ¿Qué técnica de información de retorno y corrección de errores de escritura utiliza tu profesor-a de lengua/idiomas habitualmente?:

- No corrige- Corrección explícita y/ o metalingüística -Corrección implícita (pista, código de corrección...)- Autocorrección del estudiante- Corrección entre pares

- A5- ¿En qué porcentaje crees que sabes sacar partido al uso de las TIC en el ámbito educativo? :0-25\%-25-50\%- 50-75\%-75-100\%- $100 \%$

- A6- ¿Cuántos de tus compañeros crees que saben sacar partido al uso de las TIC?: 0-25\%- $25-50 \%-50-75 \%-75-100 \%-100 \%$

- A7- ¿Deseas añadir algo más relacionado con el tema del cuestionario?

\section{REFERENCIAS}

Albirini, A. (2006). Teachers' attitudes toward information and communication technologies: The case of Syrian EFL teachers. Computers \& Education, 47(4), 373-398. https://doi 
.org/10.1016/j.compedu.2004.10.013

Alexander, B., Ashford-Rowe, K., Barajas-Murphy, N., Dobbin, G., Knott, J., McCormack, M., ... Weber, N. (2019). Horizon Report 2019 Higher Education Edition. Descargado de https://library.educause.edu/-/media/files/library/2019/4/2019horizonreport.pdf?la= en\&hash=C8E8D444AF372E705FA1BF9D4FF0DD4CC6F0FDD1

Araujo, J. C. (2013). Principales avances en el ámbito de la enseñanza de lenguas asistida por ordenador (ELAO). Ikastorratza, e-Revista de didáctica, 11, 3-92. Descargado de http://www.ehu.eus/ikastorratza/11_alea/elao.pdf

Aretio, L. G. (2021). ¿Podemos fiarnos de la evaluación en los sistemas de educación a distancia y digitales? RIED. Revista Iberoamericana De Educación a Distancia, 24(2), 9-29. https://doi.org/10.5944/ried.24.2.30223

Bax, S. (2003). CALL-past, present, and future. System, 31(1), 13-28. https://doi.org/10.1016/ S0346-251X(02)00071-4

Burston, J. (2001). Exploiting the Potential of a Computer-Based Grammar Checker in Conjunction with Self-Monitoring Strategies with Advanced Level Students of French. CALICO fournal, 18(3), 499-515. http://dx.doi.org/10.1558/cj.v18i3.499-515

Chapelle, C. A. (2007). Technology and second language acquisition. Annual Review of Applied Linguistic, 27, 98-114. https://doi.org/10.1017/S0267190508070050

Chapelle, C. A., y Sauro, S. (Eds.). (2017). The Handbook of Technology and Second Language Teaching and Learning. New Jersey: John Wiley \& Sons.

Dalton, D. W., y Hannafin, M. J. (1987). The effects of word processing on written composition. The fournal of Educational Research, 80(6), 338-342. Descargado de http://www.jstor.org/stable/27540262.

Dudeney, G., y Hockly, N. (2007). How to teach English with technology. Pearson Longman.

Ferris, D., y Roberts, B. (2001). Error feedback in L2 writing classes: How explicit does it need to be? fournal of second language writing, 10(3), 161-184. https://doi.org/10.1016/ S1060-3743(01)00039-X

Frase, L. T., Kiefer, K. E., Smith, C. R., y Fox, M. L. (1985). Theory and practice in computeraided composition. En S. W. Freedman (Ed.), The acquisition of written language (pp. 195-210). NJ: Ablex Publishing Corporation.

González-Rodríguez, L. M., y Álvarez Mosquera, P. (2020). The Impact of Feedback and Explicit Rhetorical Instruction on EFL Students' Writing Proficiency in Higher Education. Atlantis. Journal of the Spanish Association for Anglo-American Studies, 42(1), 120-142. https://doi.org/10.28914/Atlantis-2020-42.1.07

Hamel, M. J. (2010). Prototype d'un dictionnaire électronique de reformulation pour apprenants avancés de français langue seconde. Recherche et pratiques pédagogiques en langues de spécialité. Cahiers de l'Apliut, 29(1), 73-82.

Heift, T., y Vyatkina, N. (2017). Technologies for teaching and learning L2 grammar. En and others (Ed.), The handbook of technology and second language teaching and learning (pp. 26-44).

Kannan, J., y Munday, P. (2018). New trends in second language learning and teaching through the lens of ICT, networked learning, and artificial intelligence. Círculo de Lingüística Aplicada a la Comunicación, 76, 13-30. https://doi.org/10.5209/CLAC.62495

Lavilla, M. (2016). Inteligencia artificial: las tecnologías que cambiarán la educación en 2030 (and others, Ed.).

Lee, I. (2014). Feedback in writing: Issues and challenges. Assessing Writing, 19, 1-5. http:// dx.doi.org/10.1016/j.asw.2013.11.009

Lee, R. S. (1970). Social attitudes and the computer revolution. Public Opinion Quarterly, 34(1), 53-59. https://doi.org/10.1086/267772

Levy, M. (1997). Computer-assisted language learning: Context and conceptualization. Oxford University Press.

MacArthur, C. A. (2009). Reflections on research on writing and technology for struggling writers. Learning Disabilities Research \& Practice, 24(2), 93-103. https://doi.org/10.1111/ 
j.1540-5826.2009.00283.x

Mangisch, G. C., y Mangisch-Spinelli, M. D. R. (2020). El uso de dispositivos móviles como estrategia educativa en la universidad. RIED. Revista Iberoamericana de Educación a Distancia, 23(1), 201-222. https://doi.org/10.5944/ried.23.1.25065

Muehleisen, V. (1997). Projects using the Internet in college English classes. The Internet TESL journal, 3(6), 1-7. Descargado de http://iteslj.org/Lessons/Muehleisen-Projects.html

Nhu, P. T. T., Keong, T. C., y Wah, L. K. (2018). Exploring teaching English using ICT in Vietnam: The lens of activity theory. International fournal of Modern Trends in Social Sciences, 1(3), 15-29.

Pokrivcakova, S. (2019). Preparing teachers for the application of AI-powered technologies in foreign language education. Fournal of Language and Cultural Education, 7(3), 135-153. https://doi.org/10.2478/jolace-2019-0025

Puentedura, R. (2013). SAMR: Moving from Enhancement to Transformation. Descargado de http://www.hippasus.com/rrpweblog/archives/2013/05/29/ SAMREnhancementToTransformation.pdf

Romero, F., Carrió, M. L., y Yang, Q. (2016). Hábitos de consumo TIC de los estudiantes universitarios: estudio comparativo entre China y España. En A. Díez-Mediavilla, V. Brotons, D. Escandell, y J. Rovira (Eds.), Aprendizajes plurilingües y literarios: nuevos enfoques didácticos (pp. 1013-1020). Servicio de Publicaciones UPV. Descargado de http://hdl.handle.net/10045/64942

Salaberry, M. R. (2001). The use of technology for second language learning and teaching: A retrospective. The modern language journal, 85(1), 39-56. https://doi.org/10.1111/ 0026-7902.00096

Sheppard, K. (1992). Two feedback types: Do they make a difference? . RELC journal, 23(1), 103-110. https://doi.org/10.1177/003368829202300107

Thu-Dang, X. (2013). ICT in foreign language teaching in an innovative university in Vietnam: Current practices and factors affecting ICT use (Tesis Doctoral no publicada). Torrens University, Australia.

Torres, N. V. (2006). How Well are ESL Teachers Being Prepared to Integrate Technology in Their Classroom? Teaching English as Second or Foreign Language e-fournal, 9(4), 1-28.

Warschauer, M., y Kern, R. (2000a). CALL for the 21st Century. IATEFL and ESADE Conference, 2 fuly, Barcelona, Spain.

Warschauer, M., y Kern, R. (Eds.). (2000b). Network-based language teaching: Concepts and practice. Cambridge university press. https://doi.org/10.1017/CBO9781139524735

Weil, M. M., y Rosen, L. D. (1995). The psychological impact of technology from a global perspective: A study of technological sophistication and technophobia in university students from twenty-three countries. Computers in human behavior, 11(1), 95-133. https://doi.org/10.1016/0747-5632(94)00026-E

Wolfe, E. W., Bolton, S., Feltovich, B., y Bangert, A. W. (1996). A study of word processing experience and its effects on student essay writing. Fournal of educational computing research, 14(3), 269-283. https://doi.org/10.2190/XTDU-J5L2-WTPP-91W2

Young, S. S. C. (2003). Integrating ICT into second language education in a vocational high school. Journal of Computer Assisted Learning, 19(4). https://doi.org/10.1046/j.0266 -4909.2003.00049.x

Yunus, M.-M. (2007). Malaysian ESL teachers' use of ICT in their classrooms: expectations and realities. RECALL: The fournal of EUROCALL, 19(1), 79-95. http://dx.doi.org/10 .1017/S0958344007000614

Zawacki-Richter, O., Marín, V. I., Bond, M., y Gouverneur, F. (2019). Systematic review of research on artificial intelligence applications in higher education-where are the educators? International fournal of Educational Technology in Higher Education, 16(39), 39-39. https://doi.org/10.1186/s41239-019-0171-0 